\title{
Reviewers in this Issue
}

Dr. M. A. Salam Khan

Professor of Anaesthesiology, SICU

BIRDEM Academy and Hospital, Dhaka, Bangladesh

Dr. Ferdous Ara

Professor of Pharmacology

Delta Medical College, Dhaka, Bangladesh

Dr. S. M. Fazlul Karim

Professor of Biochemistry

Delta Medical College, Dhaka, Bangladesh

Dr. Md. Nur Hossain

Professor of Forensic Medicine

Delta Medical College, Dhaka, Bangladesh

Dr. Shamim Ahmed

Professor of Anatomy

Kumudini Women's Medical College, Tangail, Bangladesh

Dr. Md. Rezwanur Rahman

Professor of Biochemistry

Delta Medical College, Dhaka, Bangladesh

\section{Dr. Rehnuma Jahan}

Consultant of Gynecology \& Obstetrics

Square Hospital, Dhaka, Bangladesh

Dr. Ahmedul Kabir

Associate Professor of Medicine

Dhaka Medical College, Dhaka, Bangladesh 